\title{
COMPLEX PRICE DYNAMICS IN THE MODIFIED KALDORIAN MODEL
}

\author{
Jan Kodera, Quang Van Tran, Miloslav Vošvrda*
}

\begin{abstract}
:
In this article we analyse a neoclassical model of inflation. Our aim is to reconstruct the neoclassical theory of inflation to obtain a model which generates non-periodical oscillations of price level. This model is considered to be a realistic approximation of actual price level evolution. We start our analysis with the Fisherian equation of exchange. The assumption on non-variability of the velocity of money circulation parameter is relaxed in favour of dependence on expected inflation. The resulting model of inflation is a two-equation model where price evolution depends on production dynamics which is assumed to be an exogenous variable. After that, the two-equation model is re-formulated as an autonomous system to a model where production dynamics is determined by a Kaldorian type's model. By adding Kaldor's model to the two equation system, we create a four equation model. Both our models are able to generate more complex dynamics, i.e. non-linear cycles and chaos, which we examine by generating time series from numerical example and analyse them with the help of an advanced non-linear method.
\end{abstract}

Keywords: Price dynamics, two-equation model, four-equation model, numerical examples, non-linear time series analysis

JEL Classification: E32, E37

\section{Introduction}

The aim of this article is to provide an answer to the question whether the traditional models of inflation are able to generate more complex dynamics. As we know the actual time series of economic variables very often exhibit non-periodical oscillations. This phenomenon is in direct contradiction to the behaviour of linear dynamic models which are traditionally used for modelling economic time series. These models could only capture linear (symmetric, periodical) explosive or diminishing oscillations in these series. Though persistent oscillations are also possible in the linear system, they are only singular solutions for a given set of parameters. On that aspect, the theory of linear dynamic systems is perfectly elaborated using the very effective tools of matrix theory. Furthermore, linear dynamical models are very often disturbed by a stochastic process. In this way we obtain instead of linear oscillations irregular motion reminiscent

* Jan Kodera, Tran Van Quang, University of Economics, Prague, nám. W. Churchilla 4, 13067

Prague 3 (kodera@vse.cz); Miloslav Vošvrda, Institute of Information Theory and Automation, AS

CR, Pod Vodárenskou věží 4, 18208 Prague 8 (vosvrda@utia.cas.cz).

The support from the Czech Science Foundation under Grant P402/12/G097 is gratefully acknowledged. 
of actual motion of economic variables. Though stochastic linear models present a viable scientific method of economics and econometrics with many applications, it is unable to identify the source of irregular and endogenous fluctuations of economic variables.

Unlike linear model, non-linear deterministic systems could display non-linear, non-periodic oscillations which look like stochastic chaos. The theory of non-linear deterministic systems can be found in Guckenheimer's and Holmes' famous work (1986). Their work attracted many followers, for example Kuznetsov (1998), Perko (2001). According to this theory, an economy is a non-linear system, in which its dynamics is fully coincident with the actual motion of its variables producing non-periodic oscillations. This interpretation of economic systems is fundamental in non-linear deterministic systems because according to it the origin of economic fluctuations results from the inside structure of the system, not as a consequence of irregular external shocks as the real business cycles theory suggests.

In our article we will use the macroeconomic approach based on traditional macroeconomic theory to which non-linear relationships are added. Since non-linear models can exhibit more complex behaviour, i.e. non-periodical oscillations, we formulate a non-linear dynamical system to describe the behaviour of an economy. We begin with a two-equation model including expected inflation and a money market based on the work of Chiarella (1990). In our model, expected inflation and price level are endogenous variables while production and money supply are exogenous ones and instead of function arctan used by Chiarella for the relationship between velocity of money and expected inflation we express this relationship by a logistic function which seems to be a more appropriate choice for this purpose than function arctan.

After that, we construct another model which consists of four differential equations describing the relationships of economic variables in commodity market, capital formation, expected inflation and money market. To capture the dynamics of production and capital formation, we employ the well-known Kaldor's model (1940) which contains two equations. In this two equation system, the first one describes production dynamics and the second one describes the dynamics of capital formation. This system itself produces non-linear periodical oscillations of production and capital. Then we merge this two equation model with the modified two-equation model proposed by Chiarella (1990), so that the Kaldor's model will determine production which becomes an endogenous variable of the entire system. By this way, we create a four-equation non-linear model able to generate even more complex and chaotic oscillations.

In the empirical part of our work, firstly we will demonstrate how our models work on several numerical examples. Then we use our models to generate a univariate time series and with help of non-linear technique for time series analysis, we reconstruct the original dynamics from one dimensional time series and calculate two important measures of chaotic behaviour: the Lyapunov exponent and correlation dimension from the time series in order to verify the ability of non-linear technique to detect chaotic dynamics from time series produced by our models. The structure of the paper is as follows. In 
Section 2, we provide a short literature review on this matter. In Section 3 the presentation of the two-equation model, Kaldor's model and the four-equation model is presented. In Section 4 we proceed with the testing of time series generated by two-equation and four-equation models with help of Lyapunov exponents and correlation dimension. In Section 5 we will end with some concluding remarks.

\section{Releated Literature}

Oscillations of economic variables have become an important subject of interest for researchers since nineteen thirties. Economists have tried hard to find the cause of economic fluctuations and several theories on this matter have been developed. Until recently, the real business cycle theory is considered to be the most comprehensive theory which is capable of describing economic cycles with the highest rate of pregnancy. The theory emerged in the beginning of the eighties and the first serious work on this matter was established by Kydland and Prescott (1982). According to this theory, the original cause of economic fluctuations is any kind of external shock or disturbance, but mainly of technological character, that hits the economy and details it from the equilibrium position. The shock is spread into different variables over time due to the inter-temporal connections among them. Through market mechanism, economic variables adjust themselves to a new level and a new equilibrium is established. Therefore, economic fluctuations, resulting from these microeconomic interactions, are the best response to an external shock. The dominance of real business cycle theory has considerably suppressed the room given to macro-economic approach in order to elaborate business cycle for a long period. The prevalence of real business cycle theory is clearly seen when we compare the amount of publications somehow connected with the real business cycle theory with the quantity of publications using macroeconomic approach.

Despite the undeniable dominance of the real business cycle theory in the recent past, one can find works on economic fluctuations resulting from endogenous causes. In fact, its dominance is somehow eroded in the light of the recent economic turbulence. Those dismissive remarks of Rosser (1999) on the business cycles theory from the macroeconomic perspective might be premature and one may expect some resurrection of the macroeconomic approach in the near future. But let's start with a brief description of development of this theory from the very beginning.

The first attempt to explain the cause of economic oscillations based on macroeconomic approach can be dated back to the beginning of nineteen thirties in the works of Tinbergen (1930) and Frisch (1933) who tried to explain the cyclical behaviour of an economy through the fluctuations of investments with the help of a difference-differential equation. Then Kaldor (1940), contrary to the traditional Keynesian multiplier-accelerator concept, came up with the idea that savings and investments are not linear function of production. Thank to this non-linearity, the economy as a system can generate the well-known cyclical behaviour. Since our work is linked to this Kaldor's idea, we will come back to it 
in full details in the next section. Later, Goodwin, Samuelson, Phillips (see Allen, 1956) further developed the endogenous concept of business cycles. In principle, Samuelson's approach to how to explain business cycles is based on discrete dynamics. According to him, consumption is a linear function of delayed incomes and as income accelerates capital accumulation, investment is also a function of lagged production. From these assumptions Samuelson derives a linear difference equation of second order. For a certain combination of its parameters, this equation can generate periodic oscillations, which is the origin of fluctuations in an economy. Phillips (1954) has built his model on the same assumption, but unlike Samuelson, he uses the continuous time framework. In his model he unified the multiplier and the accelerator principle and derived a linear differential equation of second order which can produce linear oscillations. Finally, Goodwin (1951) assumes that actual realized capital stock rarely can reach the desired level with respect to product and he introduced a non-linear accelerator and studied the impact of this imbalance on business cycles. He found that without technological progress, an economy in this setting can oscillate without any exogenous factor, the equilibrium of the whole economy is unstable. However, due to the non-linearity, the economy does not explode and is kept inside a limit cycle. With technological progress, there is no equilibrium point and the depression periods of the economy are always shorter than the boom ones which is in line with the observed stylized facts on business cycles.

Kalecki (1971) has immensely contributed to the modern macroeconomic theory of economic cycles and oscillations putting it more precisely. In his work, Kalecki goes back to the idea suggested by Tinbergen and Frisch in the early nineteen thirties. According to him, in each investment one must distinguish three following stages: firstly, the time, when a decision on investment is made, then the period of time needed for the production of capital goods and finally, the time when the capital goods are delivered. In a continuous economy, given the law of motion of capital stock and with respect to the depreciation relationship of capital, by this construction, the dynamic of capital stock in the economy is a non-linear difference-differential equation. Capital stock in the model described by this difference-differential equation exhibits more complex behaviour including chaos. As a result, oscillations of capital stock induce fluctuations of other economic variables.

Chiarella (1990) takes a step further in the endogenous macroeconomic approach and proposes a continuous model in which non-linear economic dynamics is explained. His idea is closely linked to Goodwin's model of business cycle presented above. He also modifies Goodwin's model but, above all, he introduces a model of monetary dynamics with adaptive expectation of inflation. The non-linear relationship in his model is embodied in the velocity of money circulation, where the velocity is a non-linearly function of expected inflation ${ }^{1}$. Chiarella's model is improved further by Flaschel, Franke and Semmler (1997). These authors introduce a class of non-linear dynamical systems in which they examine the behaviour of monetary sector in short-run,

1 The expected inflation is defined as an annualized relative infinitesimal change in the expected price level. 
macroeconomic fluctuations as well as the interaction of product, labour, and monetary sector. These models can exhibit even more complex dynamic behaviour and the authors have substantially contributed to the further development of the theory on non-linear deterministic systems. As our work is built upon these significant contributions, we will explain them in detail in the next section.

In this short literature review we have shown that the macroeconomic approach of explaining economic fluctuations has not died as some may think. On the contrary, it still has its own followers. In our work we will make use of various ideas of our predecessors and add new suggestions to it. Namely, we replace the original fiction in Chiarella's model by a logistic function. Later we combine Kaldor's model with the modified Chiarella's model to get richer dynamics. From the mathematical point of view, we are benefited from the use of Guckenheimer's book as a broad guide to non-linear dynamical systems. We also take a huge advantage from Perko's book which takes us through when dealing with linear and especially non-linear equations and their applications in non-linear dynamical systems. Finally, we would like to mention Kuznetsov's book where the author develops an effective theory of bifurcation and its application.

\section{Two and Four-Equation Models of Price Dynamics}

\section{The two-equation model}

Let us start with a neoclassical model of money market with adaptive expectation of inflation. The model contains two equations. The first equation in this two-equation model of price dynamics is based on the equation of exchange or the Fisherian equation:

$$
M V_{T}=P T,
$$

where $M, V_{T}, P, T$ denote the money supply, transactions velocity of money circulation ${ }^{2}$, the price level ${ }^{3}$ and the number of trade (real transactions) respectively. The supply of money is considered to be an exogenous variable. The equation of exchange is usually expressed in the production form. Let $Y$ be a final production (gross domestic product) and let us put

$$
a=\frac{Y}{T} .
$$

Then equation (1) gets the form

$$
M V=P Y,
$$

2 Transactions velocity of money circulation is a number of transactions carried out by one money unit in a time period.

3 The price level is defined as the changes in the prices of market goods over time with respect to the prices in the base period. 
where $V=a V_{T}$. The production form of the exchange equation is more convenient for our analysis. Note that Fisherian equation (1) is not the accounting identity, but it is an equilibrium condition.

In order to present and precisely describe neoclassical theory of price movement, it is necessary to take into account not only the assumption about the nature of the equilibrium of the analysed equation, but also the assumption that $Y$ and $V$ are constant in the short run. It leads to a very simple conclusion that immediately follows from equation (2). This conclusion is that price depends on $M$, which is usually formalised by the following equation

$$
P=\frac{V}{Y} M
$$

Assuming that $V, Y$ are constant, we get a linear dependence of price level on money supply.

The processes which equalize the flow of money $M V$ with the flow of nominal final production $P Y$ are called as Marshalian or Ricardian adaptation processes. We will formulate the Marshalian adaptation process because it captures the further development of the modern neoclassical theory of inflation better. The Marshalian process states that when the system is not in equilibrium, i.e. equation (2) is not fulfilled, than there are forces which adapt the system to equilibrium through the processes acting on commodity market, for example if the supply of money is relatively high, which means

$$
M V>P Y,
$$

then the relatively high balances on current deposits are leading up to higher purchases in the commodity market. As a consequence, the price level on commodity market goes up. It means that the right hand-side of the above-mentioned equation increases and the system returns back to equilibrium.

When the supply of money is relatively low, which means

$$
M V<P Y,
$$

then economic agents start to save more money to increase relatively low balances. This reduces demand for goods and services at commodity market and hence, the price level must go down. The right-hand side keeps decreasing until the equilibrium is restored. This approach where price dynamics is influenced by money stock is applied relatively frequently, even in econometric models (see Arlt, Kodera, Mandel and Tomšík, 2006).

The modern approach to the equilibrium in money market differs from the traditional one. Equation (2) is rearranged in the following way

$$
M=\frac{1}{V} P Y .
$$


Now we define the amount of money required by agents. This quantity of money reacts to determining parameters, i.e. the price level, the product and the constant (or slowly changing and technologically determined) velocity of money. We call this quantity a demand for money and define it in the following way

$$
M^{d}=\frac{1}{V} P Y .
$$

With regard to (3), the equilibrium condition (2) has a form

$$
M=M^{d} .
$$

The traditional neoclassical theory assumes the velocity of money to be constant. Friedman in his seminal work (1956) showed the expected inflation plays an important role in money demand determination. He suggested that money demand decreases as expected inflation increases. Friedman also interpreted the decline of money demand as the traditional Fisherian theory. A more exact interpretation of the dependence of money demand on expected inflation could be found in Chiarella (1990). In his work he postulates money demand as a non-linear function depending upon expected inflation. He uses the Fisherian equation (3), where the velocity of money $V$ is an increasing function of expected inflation on the interval $(-\infty, \infty)$ and is restricted in this interval by its least upper bound and highest lower bound. For this purpose function arctan is used in Chiarella's work. This function expresses the fact that velocity of money increases in expected inflation within the bounds of technological restrictions. In our article we follow his approach but we deviate from it in details. We also consider the Fisherian money demand, but postulate the velocity of money as a logistic function of expected inflation. In our opinion the velocity function should approximate very closely highest lower bound for low inflation. Then, for increasing inflation the velocity of money increases very quickly and very quickly approximate its minimum upper bound. The logistic density function is very similar to the arctan density function and it has lighter tails than the ones of the arctan density function. In our opinion, logistic function is a more proper choice for the model. On the one hand, it is an elementary function, therefore it is easy to operate with. On the other hand, due to its nature mentioned above, it more precisely reflects the dynamics of propensity to invest in our model.

The second equation in the model which describes inflation expectation dynamics is formed adaptively. Let us assume the velocity of money in the demand for money to be an increasing function of expected inflation.

$$
M^{d}=\frac{1}{V(\pi)} P Y .
$$

where $M^{d}$ stands for demand for money, $P$ means the price level, $V$ is the velocity of money, $\pi$ denotes the expected inflation. Taking the logarithm of the above equation we get

$$
m^{d}=p+y-v(\pi)
$$


where $m^{d}$ - logarithm of demand for money, $p$ - logarithm of price level, $y$-logarithm of production, $v$ - logarithm of the velocity of money. Logarithm of the velocity of money is assumed to be given by the following equation

$$
v(\pi)=v_{0}+\kappa \cdot \theta(\pi)
$$

where constant $v_{0}$ is determined by a technological level of the banking sector. Parameter $\kappa$ is a constant and $\theta(\cdot)$ is a logistic function solving the logistic equation

$$
\frac{\mathrm{d} \theta(\pi)}{\mathrm{d} \pi}=\theta(\pi) \cdot(g-h \cdot \theta(\pi))
$$

where $g$ and $h$ are parameters. With an initial condition $\theta(0)=\theta_{0}$ we get a particular solution of the above differential equation:

$$
\theta(\pi)=\frac{g \cdot \theta_{0}}{h \cdot \theta_{0}+\left(g-h \cdot \theta_{0}\right) \cdot e^{-g \cdot \pi}} .
$$

Now we are ready to introduce a differential equation which captures the development of the price level over time in the extended model. This development is usually called as the price level dynamics.

Let us consider that variables $y, p$ and $\pi$ are smooth functions of time. The rate of inflation or an instant increment of the logarithm of price level $\dot{p}$ is affected by the disequilibrium in money market equal the difference between the constant supply of money $m$, and the demand for money $m^{d}$, so we get

$$
\dot{p}=\sigma \cdot\left(m-m^{d}\right) .
$$

where $\sigma>0$ is an adjustment parameter and $\dot{p}$ is the derivative of the logarithm of the price level with respect to time. Replacing $m^{d}$ from (4) and using (5), we get

$$
\dot{p}=\sigma \cdot\left[m-p-y+v_{0}+\kappa \cdot \theta(\pi)\right] .
$$

The above equation describes price level dynamics, which is realised through the Marshall adaptation process.

The instant increment of expected inflation is denoted by $\dot{\pi}$ and the acceleration of expected inflation is labelled by $\ddot{\pi}$. An adaptive expected inflation is expressed by

$$
\dot{\pi}=\omega \cdot(\dot{p}-\pi) .
$$

Substituting from (7) to (8) we get

$$
\dot{\pi}=\omega \cdot\left\{\sigma \cdot\left[m-p-y+v_{0}+\kappa \cdot \theta(\pi)\right]-\pi\right\} .
$$


Using this relation we get the final form of the equation describing adaptive expectation of inflation. Let us differentiate both sides of the equation (9) and replace $\dot{p}$ from equation (8). After some rearrangements we get

$$
\frac{1}{\sigma \omega} \ddot{\pi}+\left[\frac{1}{\sigma}+\frac{1}{\omega}-\kappa \frac{\mathrm{d} \theta(\pi)}{\mathrm{d} \pi}\right] \dot{\pi}+\pi=\dot{m}^{s}-\dot{y} .
$$

quation (10) is a second order differential equation for variable $\pi$ with endogenous variables $m$ and $y$. The right hand side of this equation is $\dot{m}-\dot{y}$ which equals the difference between the rate of growth of money stock and production. Let us assume that in an economy the rate of growth of production oscillates with linear periodical oscillations, for example $\dot{y}=\rho \cos t$. If the central bank decides and is able to control the money supply so that it closely follows the evolution of production growth rate, then the time dependence of growth rate of money supply is the same, thus $\dot{m}=\rho \cos t$, and therefore the right hand side of (10) equals zero.

$$
\frac{1}{\sigma \omega} \ddot{\pi}+\left[\frac{1}{\sigma}+\frac{1}{\omega}-\kappa \frac{\mathrm{d} \theta(\pi)}{\mathrm{d} \pi}\right] \dot{\pi}+\pi=0 .
$$

This example is the case when central bank chooses the monetary policy which is perfectly adaptable to production dynamics. Now, let us assume that central bank does not adapt to money supply at all. In this case money supply is a constant, therefore we have $\dot{m}=0$. Replacing this term in (10) we get

$$
\frac{1}{\sigma \omega} \ddot{\pi}+\left[\frac{1}{\sigma}+\frac{1}{\omega}-\kappa \frac{\mathrm{d} \theta(\pi)}{\mathrm{d} \pi}\right] \dot{\pi}+\pi=-\rho \cos (t) .
$$

The system of two non-linear first order differential equations was transformed into one second order differential equation. As we are dealing with a non-linear problem we have to resort to numerical methods in our analysis. Therefore we will select a set of numerical values for the parameters appeared in the above equations.

\section{Numerical example 1}

The selection of numerical values of parameters in equations (8) and (9) is submitted to the aim of this paper which is to show that non-linear dynamic models presented here are able to generate chaos. For this purpose we choose such numerical values of parameters by which the chaotic dynamics can be ensured. At the same time, we try our best to get clear economic interpretation from these parameters and get them fall in line with the underlying economic nature. Parameters of adaptation $\omega, \sigma$ are assigned values $\sigma=0.6$ and $\omega=0.8$ and the only criterion of doing so is their ability to generate chaos. If we look at equation (11) or (12) we notice that the numerical values of parameters are interchangeable without any consequence for the solution. Though the speed of adaptation in the money market and the speed of adaptation in adaptive expectation 
of inflation separately do not have significant impact on the shape of the solution of equations (11) and (12), but in combination they do influence the dynamics. Their joint effect is expressed as the product of $\sigma \omega$ and the sum $\omega^{-1}+\sigma^{-1}$.

When selecting the value for the parameters of function $\theta$ we face a similar problem. Given our primary objective in this paper as we have already stated above, we will not experiment economy with various sets of parameters. Rather we put our emphasis on keeping the shape of function in line with our belief. Calibrating the parameters and finding their values and their exact correspondence in order to make them be in accordance with economic theory will be the next stage of our research. For this reason, the parameters in the function $\theta$ are chosen as follows: $g=h=1, \theta_{0}=0.5, \kappa=15$. The value for parameter of production growth rate is chosen $\rho=0.8$.

Equation (12) becomes:

$$
\frac{1}{0.48} \ddot{\pi}+\left[\frac{1}{0.6}+\frac{1}{0.8}-15 \frac{\mathrm{d} \theta(\pi)}{\mathrm{d} \pi}\right] \dot{\pi}+\pi=-0.8 \cos (t),
$$

where

$$
\theta(\pi)=\frac{g \cdot \theta_{0}}{h \cdot \theta_{0}+\left(g-h \cdot \theta_{0}\right) \cdot e^{-g \cdot \pi}}=\frac{1}{1+e^{-\pi}} .
$$

The most effective way for obtaining the numerical solution is to transform the equation (13) into a system of three autonomous differential equations of variables $\pi, q$ and $t$. Let these variables are dependent on time denoted by $\tau$. As equation (13) is a differential equation of higher order, we transform it into a system of three differential equations of the first order to obtain its numerical solution. For this purpose, we introduce auxiliary variables $q$ and $t$ which are functions of time denoted as $\tau$. Let us remind that variable $q$ has no exact economic interpretation and define it as follows:

$$
\dot{q}=\frac{1}{0.48} \ddot{\pi}+\left[\frac{1}{0.6}+\frac{1}{0.8}-15 \frac{\mathrm{d} \theta(\pi)}{\mathrm{d} \pi}\right] \dot{\pi},
$$

Then, after integration and small rearrangement of (15), we get

$$
\dot{\pi}=0.48 q-(0.8+0.6) \pi+6.2 \theta(\pi) .
$$

Using (14) we obtain

$$
\dot{\pi}=0.48 q-(0.6+0.8) \pi+\frac{6.2}{1+e^{-\pi}} .
$$

From (13) and (15) we get

$$
\dot{q}=-[\pi+0.8 \cos (t)] .
$$

The third variable of our three-equation system is time which is defined as $t=\tau$, therefore we have:

$$
\dot{t}=1
$$


We use software package Mathematica to solve this three-equation dynamical system. Phase portrait and time evolution of expected inflation $\pi$ of the above system is presented in Figures 1 and 2. In Figure 1, the phase portrait of the three-equation system is shown and we can observe its chaotic behaviour. In Figure 2, the one-dimensional evolution of expected inflation is shown and we can clearly see the non-periodical feature of its fluctuations.

Figure 1

\section{Chaotic Attractor ${ }^{4}$ for the Numerical Example 1}

Figure 2

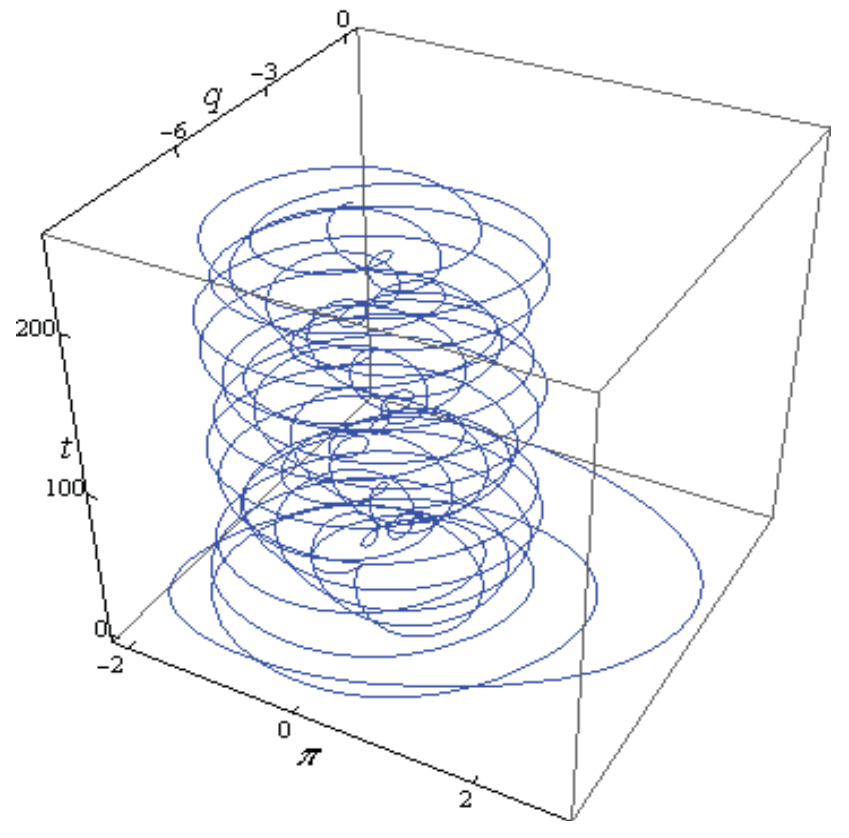

The Dynamics of the Expected Inflation $\pi$

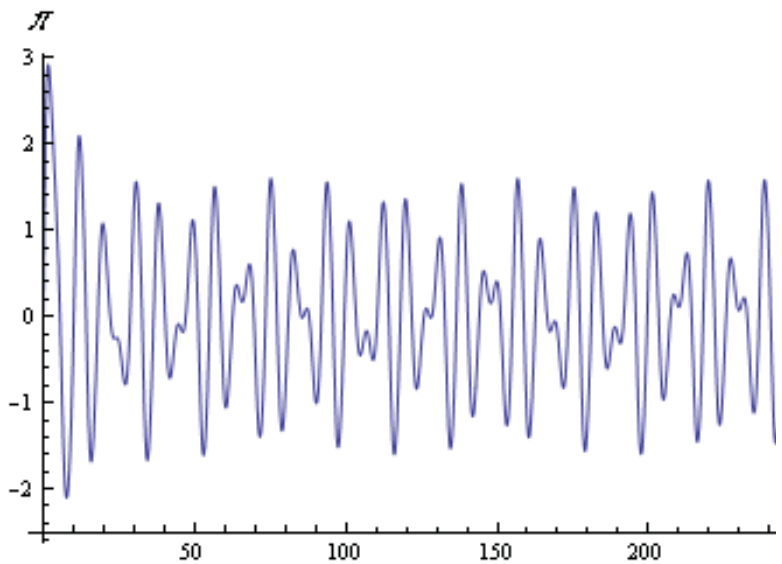

4 An attractor is a subset of space to which the initial evolution of a dynamical system is attracted eventually. 


\section{The four-equation model}

When deriving this model, we are inspired by Kaldor's seminal work (1940) which has laid an important foundation for endogenous theory of business cycle. Kaldor's model is frequently presented in many works on modern business cycle theory, for example, Gabisch and Lorenz (1989); Lorenz (1994) and Franke, Semmler (1997). These eminent works provide an analysis and further upgrading of Kaldor's model. In our work we improve the traditional Kaldor's model, where investment function will be a logistic function of productivity of capital. The two-equation model includes variable $y$, which stands for the logarithm of production. This variable is present in the right hand side of equation (7). In the original model, it is an exogenous variable and its dynamic is described by relationship $\dot{y}=\rho \cos t$ which after integration gets the form $\dot{y}=\rho \sin t$. Now we build four-equation model which includes two-equation model of price dynamics which is identical with two-equation model presented above and Kaldor's model which generates production and capital dynamics. The four-equation model contains four variables price level, expected inflation, production and capital. The dynamics in this model is endogenous.

A traditional form of this model describes the dynamics of real production and capital stock. The dynamic of production is described by

$$
\dot{Y}=\alpha[I(Y, K)-S(Y)]
$$

where $Y, K$ depend on time and stand for a production and a capital stock respectively. The parameter $\alpha>0$ is the adjustment parameter. Investments $I$ are increasing in $Y$ and are decreasing in $K$. The savings $S$ are an increasing function of $Y$. The equation (16) describes a production dynamics which is expressed as a consequence of disequilibrium between investments and savings.

The capital increase $\dot{K}$ is equal to the difference of investment and capital consumption. The capital consumption is assumed to be an increasing function of capital stock $D(K)$. Hence we can formalize the equation expressing capital dynamics as follows

$$
\dot{K}=I(Y, K)-D(K)
$$

The investment function is supposed to be the product of a propensity to invest $j=\frac{I}{Y}$ which depends on an expected productivity of capital $\chi$ and production $Y$

$$
I(Y, K)=j(\chi) Y .
$$

Assuming that agents are expecting actual product of capital, we have

$$
\chi=\frac{Y}{K} \text {. }
$$

With regard to the above relation, the equation (17) gets a form

$$
I(Y, K)=j\left(\frac{Y}{K}\right) Y=j\left(e^{y-k}\right) Y=i(y-k) Y
$$


From now on we will use logarithmic expressions. Let's denote $\varepsilon=\log \chi=y-k$, where $y, k$ stand for $y=\log Y, k=\log K$ respectively. Using this notation we can transform (17) as follows:

Function $i$ is a composite function of $y-k$ on domain $(-\infty, \infty)$, which captures the dependence of propensity to invest on logarithm of $Y / K$. The propensity to invest $i$ is an increasing function of $y-k$ and is assumed to approach zero as $\varepsilon$ decreases to minus infinity and approaches to the maximum level for increasing $y-k$. We assume that a product of a constant $\mu$ and a logistic function $\lambda$, depending on $\varepsilon$, is a reasonable approximation of the propensity to invest. The logistic function $\lambda$ is the solution of the following differential equation

$$
\frac{\mathrm{d} \lambda(\varepsilon)}{\mathrm{d} \varepsilon}=\lambda(\varepsilon) \cdot(a-b \cdot \lambda(\varepsilon)) .
$$

Let us consider an initial condition $\lambda(0)=\lambda_{0}$. Then the logistic function $\lambda$ takes on the following form

$$
\lambda(\varepsilon)=\frac{a \cdot \lambda_{0}}{\left.b \cdot \lambda_{0}+\left(a-b \cdot \lambda_{0}\right)\right) \cdot e^{-a \cdot \varepsilon}} .
$$

We assume the propensity to invest to be a product of an arbitrary positive constant $\mu$ and function $\lambda$

$$
i(\varepsilon)=\mu \cdot \lambda(\varepsilon)=\frac{a \cdot \mu \cdot \lambda_{0}}{b \cdot \lambda_{0}+\left(a-b \cdot \lambda_{0}\right) \cdot e^{-a \cdot \varepsilon}} .
$$

or

$$
i(y-k)=\mu \cdot \lambda(y-k)=\frac{a \cdot \mu \cdot \lambda_{0}}{b \cdot \lambda_{0}+\left(a-b \cdot \lambda_{0}\right) \cdot e^{-a \cdot(y-k)}} .
$$

For the saving function we are going to use the following expression

$$
S(Y)=\left(s_{0}+s_{1} \cdot y\right) \cdot Y .
$$

The above equation describes the dependence of savings on investments as the product of a production $Y$ and a propensity to save $s_{0}+s_{1} \cdot y$ which depends on $y$. Let us rearrange equation (17) using the expressions for investments and savings. We get

$$
\dot{Y}=\alpha \cdot\left[i(y-k) \cdot Y-\left(s_{0}+s_{1} \cdot y\right) \cdot Y\right] .
$$

Dividing equation (18) by $Y$, we obtain

$$
\dot{y}=\alpha \cdot\left[i(y-k)-\left(s_{0}+s_{1} \cdot y\right)\right] .
$$

Let $D=\beta \cdot K^{\gamma}, \beta, \gamma \in(0,1)$ denote a capital consumption expressing the depreciated portion of capital. The capital formation in the closed economy is described by the following differential equation

$$
\dot{K}=I(Y, K)-\beta \cdot K^{\gamma},
$$


which can be expressed in the following form:

$$
\dot{K}=i(y-k) \cdot Y-\beta \cdot K^{\gamma}
$$

Dividing the above equation by $K$ we get

$$
\frac{\dot{K}}{K}=i(y-k) \cdot \frac{Y}{K}-\beta \cdot K^{\gamma-1} .
$$

Using logarithms instead of original values of $Y$ and $K$ we get

$$
\dot{k}=i(y-k) \cdot e^{y-k}-\beta \cdot e^{(\gamma-1) \cdot k} .
$$

Equations (19) and (20) describe the production dynamics and the capital formation. The final forms of these equations are as follows

$$
\begin{aligned}
& \dot{y}=\alpha\left[\frac{a \cdot \mu \cdot \lambda_{0}}{b \cdot \lambda_{0}+\left(a-b \cdot \lambda_{0}\right) e^{-a(y-k)}}-\left(s_{0}+s_{1} y\right)\right] . \\
& \dot{k}=\frac{a \cdot \mu \cdot \lambda_{0}}{b \cdot \lambda_{0}+\left(a-b \cdot \lambda_{0}\right) \cdot e^{-a(y-k)}} \cdot e^{y-k}-\beta \cdot e^{(\gamma-1) k}
\end{aligned}
$$

Now we will present four-equation model of price dynamics. In fact, this model is very similar to the two equation model but the external linear oscillation of the rate of product growth $(\rho \cos \dot{y})$ is replaced by the non-linear oscillation generated by the system of equations (21) and (22) which are the equations constituting specific type of Kaldor's model. Some preliminary analysis of four-equation model was established in works of Kodera and Vošvrda (2006) and Kodera (2002) where the four-equation model was drafted and its ability to generate chaotic dynamics was shown.

Taking into account equations (6), (7) and (8) we get

$$
\begin{gathered}
\dot{p}=\sigma \cdot\left(m-y-p+v_{0}+\kappa \frac{g \cdot \theta_{0}}{h \cdot \theta_{0}+\left(g-h \theta_{0}\right) \cdot e^{-g \cdot \pi}}\right) \\
\dot{\pi}=\omega \cdot\left[\sigma \cdot\left(m-y-p+v_{0}+\kappa \frac{g \cdot \theta_{0}}{h \cdot \theta_{0}+\left(g-h \cdot \theta_{0}\right) \cdot e^{-g \cdot \pi}}\right)-\pi\right] .
\end{gathered}
$$

Now we have equations (21) and (22) for real segment of economy and equations (23) and (24) for its monetary segment. Together they constitute an autonomous system of economy with complex dynamics of price level and expected inflation. The following simple numerical example gives a possible evolution of prices and inflation expectation.

The structure of the above four-equation system has interesting features. The system of equations (21) and (22) influences the system of two equations (23) and (24), but it is not 
reversely influenced by that system, because neither $p$ nor $\pi$ is present in this system. The system of equations (21) and (22) supplies the system of equations (23) and (24) with periodically oscillating variable $y$, which produces the chaotic oscillations of variables $p$ and $\pi$ in the system of equations (23) and (24) as we will see later.

\section{Numerical example 2}

Let $\alpha=35, \mathrm{a}=b=1, \lambda_{0}=1 / 2, \mu=0.25, s_{0}=0.2, s_{1}=0.05, s_{2}=0, \beta=0.1, \gamma=1$. The numerical values for monetary segment are the following: $\sigma=0.6, \omega=0.8, m^{s}=2, v_{0}=1, g=h=1$, $\theta_{0}=0.5, \kappa=30$. Equations (21)-(24) become:

$$
\begin{gathered}
\dot{y}=35\left[\frac{0.25}{1+e^{-(y-k)}}-(0.2+0.05 y)\right] \\
\dot{k}=\frac{0.25}{1+e^{-(y-k)}} e^{y-k}-0.1 \\
\dot{p}=0.6\left(2-y-p+1+\frac{15}{1+e^{-\pi}}\right) \\
\dot{\pi}=0.8\left[0.6\left(2-y-p+1+\frac{15}{1+e^{-\pi}}\right)-\pi\right]
\end{gathered}
$$

The first and the second equation could be solved separately as the system of two variables $y, k$. In this case the system exhibits limit cycle as it is shown in Figure 3. The periodical evolution of variables $y, k$ can be found in Figure 4. We can observe non-linear and periodical behaviour of the two variables of production and capital. We can also observe higher fluctuations of production compared to the ones of capital. Further, in the dynamics of capital, the slower decay alternates with its faster growth.

Figure 3

The Phase Portrait of Kaldor's Model

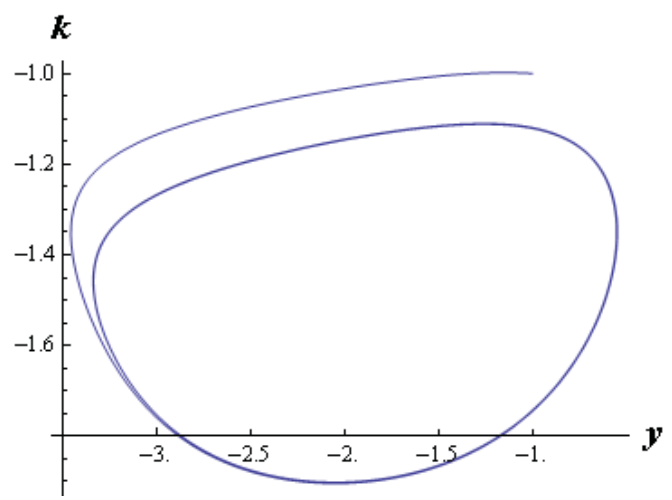


Figure 4

The Evolution of Variables $y$ and $\boldsymbol{k}$ (dashed line)

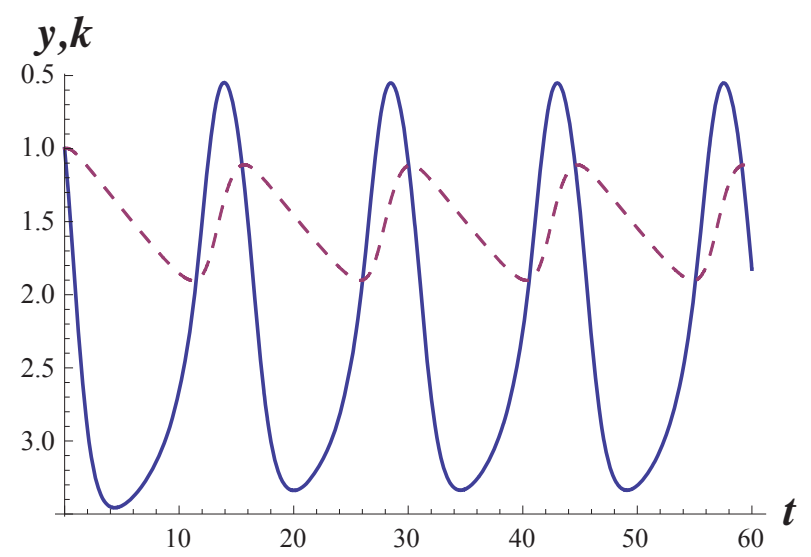

The behaviour of the above four equation system is shown in Figures 5 and 6. In Figure 5 we show the projection of attractor in the three dimensional space of axis $y, p, \pi$. The evolution of the variable $\pi$ is shown in Figure 6 .

Figure 5

The Projection of Attractor into Space $y, p, \pi$

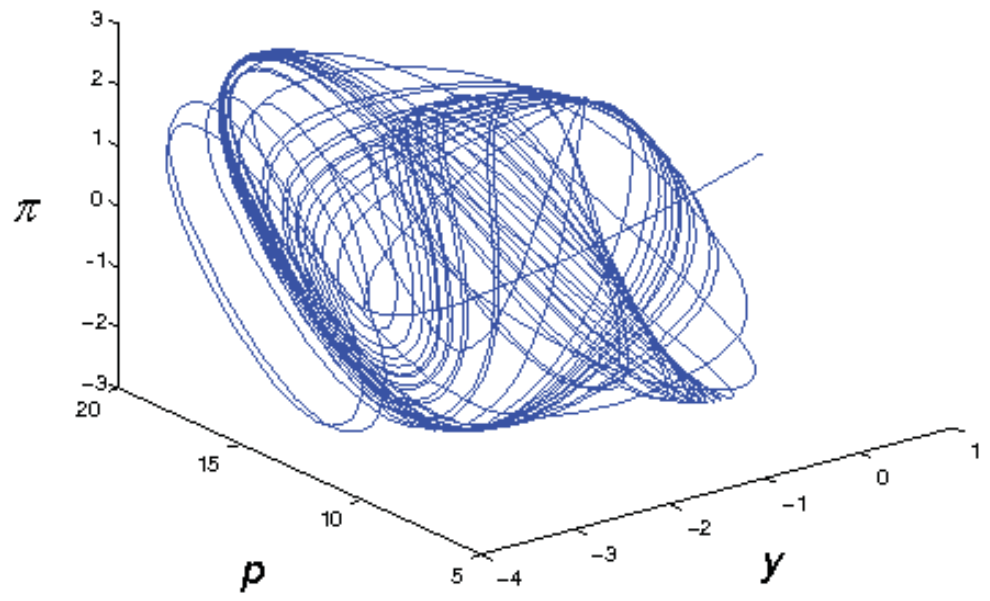




\section{Figure 6}

The Evolution of Expected Inflation

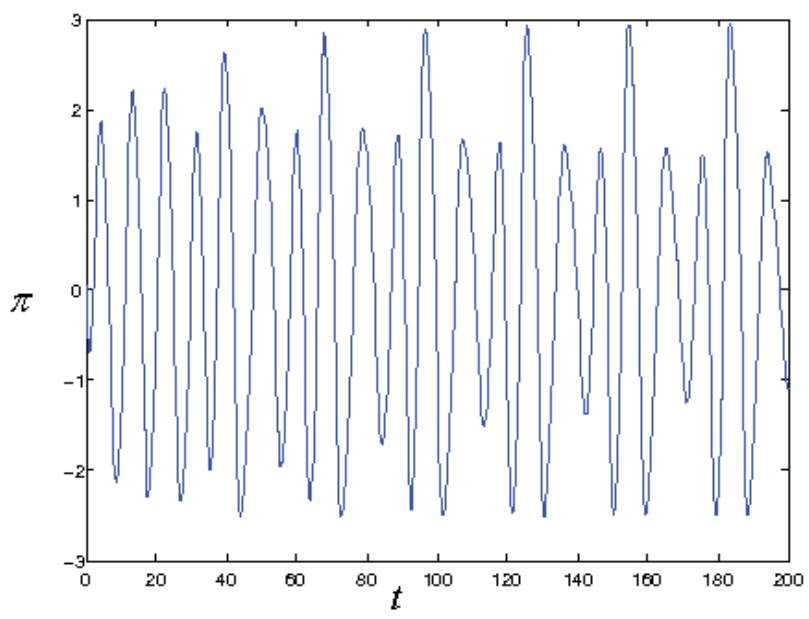

In this section, we have drafted two different models intended to explain the complex dynamics of price level series. Both models exhibit non-periodic oscillations but there is some dissimilarity between them. The two-equation model consists of equations (7) and (8) for the price dynamics, in the two-equation model dynamics of price level and expected inflation depends on the difference between rates of growth of its variables, see equation (10). If $y$ and $m$ oscillates and the linear oscillations (i.e. functions sine or cosine) have sufficiently large amplitude, then the price dynamics of this model could be chaotic (see Chiarella, 1990). If the difference between rates of growth of variables $y$ and $m^{s}$ are small or even zero, then the chaotic movements in price dynamics diminishes and it is replaced by non-linear periodical oscillations. The two equation model is able to produce chaotic oscillations but its disadvantage is that the dynamics of the variable $y$ (and $m^{s}$ ) is determined outside the system. The system works as a forced oscillator.

The four-equation model consists of two equations (7) and (8) describing the price dynamics and two other equations (19) and (20) capturing the dynamics of production and capital. This dynamics is represented by Kaldor's model. As we know, Kaldor's model is a relatively closed one whose variables are not affected by variables present in the two-equation model for the price dynamics. On the other hand, the model of price dynamics does contain one variable which is production $y$ from Kaldor's model. By linking them together, we examine how fluctuations in the real sector affect the price dynamics in the original two-equation model. It turns out that the (four-equation) model could produce chaotic oscillations as well. We have found that both can produce chaotic dynamics. In the next section, we will examine how the characteristics of the chaos of two-equation model differ from the ones of the four-equation model. 


\section{The Analysis of Time Series}

In this section we will analyse the evolution of price level and expected inflation. The evolution of these two variables is represented by time series which are generated by the two equation model calibrated in numerical example 1 in Section 3. Time series of our interest will be examined in state space in such a way that first we reconstruct their trajectories in state space and then the chaotic descriptors will be estimated as recommended by Kantz (1997) and Sprott (2003).

For this purpose the correlation dimension and the largest Lyapunov exponent are used. For better understanding let us make a brief digression to remind the reader the relevant theory. Correlation dimension and the largest Lyapunov exponent are two typical descriptors of chaotic systems, where the former describes geometrical structure of trajectories of a chaotic system in state space and the latter characterizes dynamics of the chaotic behaviour.

\section{Correlation dimension}

The geometry of a set formed by trajectories of a dynamic system in phase space often is very complicated and does not have to be an integer. On the contrary, it tends to have a fractal dimension and the existence of a fractal dimension might indicate the presence of chaos. There are many different measures for fractal dimension and one of them is correlation dimension. The calculation of correlation dimension is based on correlation integral $C$ which is defined to be the portion of pairs of points $x_{t}$ and $x_{s}$, whose distance is less than $\varepsilon$ :

$$
C(N, m, \varepsilon):=\frac{2}{N(N-1)} \sum_{m \leq t \leq s \leq N}^{N} H\left(\varepsilon-\left\|x_{t}-x_{s}\right\|\right)
$$

where $H$ is Heaviside function, $m$ is embedding dimension, and $\mathrm{N}$ is the number of observations. In the limit of an infinite amount of data $(N \rightarrow \infty)$, and for a small $\varepsilon$, one can expect the correlation sum $C$ to follow the power law $\left(C \approx e^{D}\right)$, therefore correlation dimension $D_{c}^{m}$ can be defined as the slope of the plot of $\log C(N, m, \varepsilon)$ versus $\log \varepsilon$ :

$$
D_{c}^{m}:=\lim _{\varepsilon \rightarrow 0} \lim _{N \rightarrow \infty} \frac{\log C(N, m, \varepsilon)}{\log \varepsilon}
$$

The correlation sums for the series of price level and expected inflation are calculated with program TISEAN (Hegger, 1999) for embedding dimensions up to 10. The numerical calculation of correlation dimension according to equation (25) requires $\varepsilon \rightarrow 0$ and $N \rightarrow \infty$. In reality, we have only a limited number of observations; in fact, there are only 2,500 observations of both series of price level and expected inflation. So we can evaluate correlation dimension at certain small distances and this makes correlation dimension fluctuate (seen Figures 7 and 8). In these figures, the correlation dimension of two time series of our interest are shown in various embedding dimensions. Because of 
its fluctuation, we cannot estimate a single value for correlation dimension in the whole interval, but we can only calculate a scaling region (Kantz, 1997), where the value is approximately constant. As it is seen on Figures 7 and 8 , these intervals are $(-2,1.5)$ for series of price level and $(-1.3,2)$ for series of expected inflation. It can be observed that they fluctuate inside an interval of $(2,3)$ and from these interval, the exact values of correlation dimension of series price level and expected inflation generated by the two-equation model lie in interval $(2,3)$ and they are 2.50 and 2.80 , respectively. These values indicate that the structure of the system is a fractal dimension type.

Figure 7

Correlation Dimension vs. Log $(\varepsilon)$ of Price Level Series

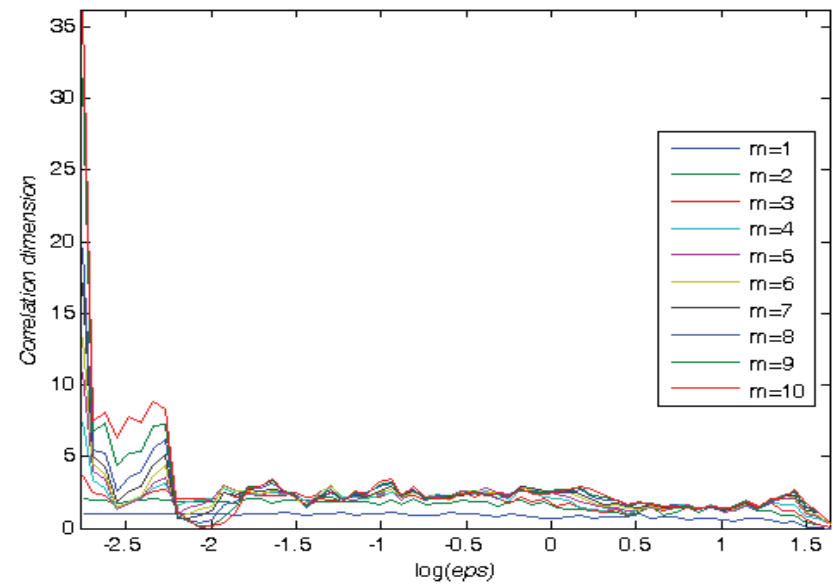

Figure 8

Correlation Dimension vs. Log $(\varepsilon)$ of Expected Inflation Series

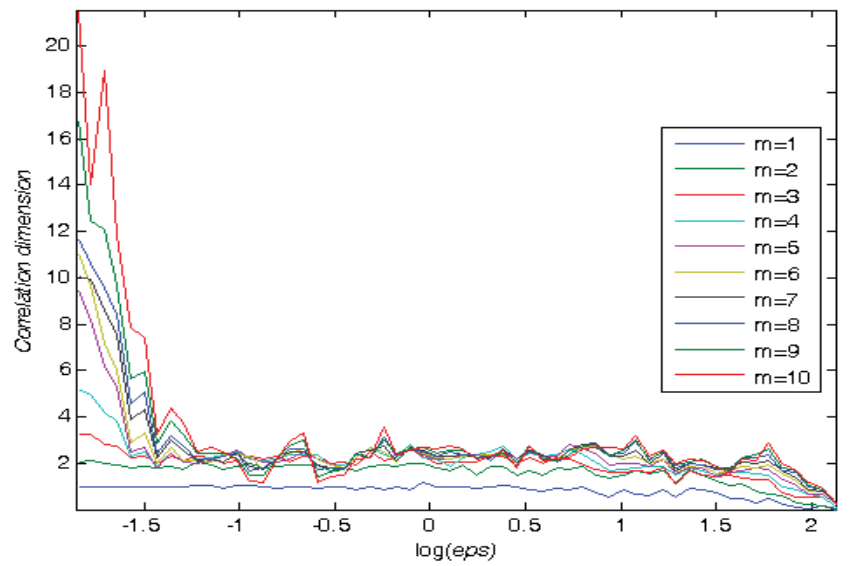




\section{The largest Lyapunov exponent}

Correlation dimension is not the only invariant that describes a chaotic system. There are other ones that are very useful and are frequently used to characterize properties of chaotic systems - such as Lyapunov exponents. Generally, they are defined as a set of logarithms of absolute values of eigenvalues of linearized dynamics of a system, averaged over the whole attractor. This means that in the phase space there is a system of eigenvectors where one certain Lyapunov exponent belongs to each of them. If at a certain moment in an $n$-dimensional space, an $n$-dimensional infinitesimal sphere containing the certain number of points lying on its surface is chosen, then this sphere can be deformed into an ellipsoid over the course of time. The measure of how much the sphere is deformed in each individual axis is dependent on the values of the corresponding Lyapunov exponents.

The estimation of all Lyapunov exponents is very difficult. For practical purposes the largest Lyapunov exponent (denoted as $\lambda$ ) is the most important one. It determines the average rate of divergence of two neighbouring trajectories starting from two close points in the state space. This divergence is an exponential process. If we choose such two points $x_{i}\left(t_{0}\right)$ and $x_{j}\left(t_{0}\right)$ in the state space at time $t_{0}$, then at time $t+t_{0}$, their distance during the course of time can be described as

$$
\left\|x_{i}\left(t+t_{0}\right)-x_{j}\left(t+t_{0}\right)\right\| \approx\left\|x_{i}\left(t_{0}\right)-x_{j}\left(t_{0}\right)\right\| e^{\lambda t} .
$$

The largest Lyapunov exponent is estimated as follows: in the state space we randomly select a set of $M$ reference states $x_{k}\left(t_{0}\right)$, for $k=1, \ldots, M$ at time $t_{0}$. Then we define a small surrounding $O_{k}$ of size $\varepsilon$ consisting of states $x_{i}^{(k)}$, for $k=1, \ldots,\left|O_{k}\right|$ to each of these reference states. Then we track how the distance between reference states $x_{k}$ and elements, which are parts of their surroundings at time $t_{0}$, will be changing over the course of time. Finally we define this variable:

$$
S(t)=\frac{1}{M} \sum_{k=1}^{M} \frac{1}{\left|O_{k}\right|} \ln \sum_{i=1}^{\left|O_{k}\right|}\left\|x_{k}\left(t+t_{0}\right)-x_{i}^{(k)}\left(t+t_{0}\right)\right\| .
$$

Needless to mention that if two close trajectories diverge from each other in a state space, then $S(t)$ grows when $t$ increases. The slope of the increasing part of the plot of relation $S(t)$ versus $t$ is the value of the largest Lyapunov exponent. All known states in the state space can gradually be selected as reference states and the sizes of $\varepsilon$ and $O_{k}$ should be chosen to be large enough so that each reference point has several of its own neighbours (Horák et al., 2003).

To calculate the largest Lyapunov exponent in our price level and expected inflation series two important parameters need to be determined first: time delay and embedding dimension $m$. To avoid information redundancy, the value of the time delay for price level and expected inflation series is chosen as 17 and 19 respectively, where the first minimum of mutual information occurs. As far as the value of embedding dimension is 
concerned, we follow the rule suggested by Takens (1985) which states that $m$ should be greater than $2 D_{c}^{m}$. Because $D_{c}^{m}<3$ as it was mentioned before, the largest Lyapunov exponent will be estimated for $m=2$ to 7 . The values of $S(t)$ are calculated by TISEAN software package again. The plots of $S(t)$ versus $t$ for series of price level and expected inflation are shown in Figures 9 and 10. In both figures, before reaching the area of saturation, an area of linear growth, can be seen in the course of time. The slopes of these areas decrease rapidly with increasing correlation dimension. As mentioned before, the largest Lyapunov exponent is the slope of the area with linear growth with lower embedding dimension. The values of the largest Lyapunov exponent calculated by this method for series of price level and expected inflation are rather small and are 0.08 and 0.02 , respectively. These numbers show that the dynamics of the system of two equations can be reliably forecast in intervals $(0,0.08)$ and $(0,0.02)$ of time.

Figure 9

The Plot of $S(t)$ versus Time of Price Level Series

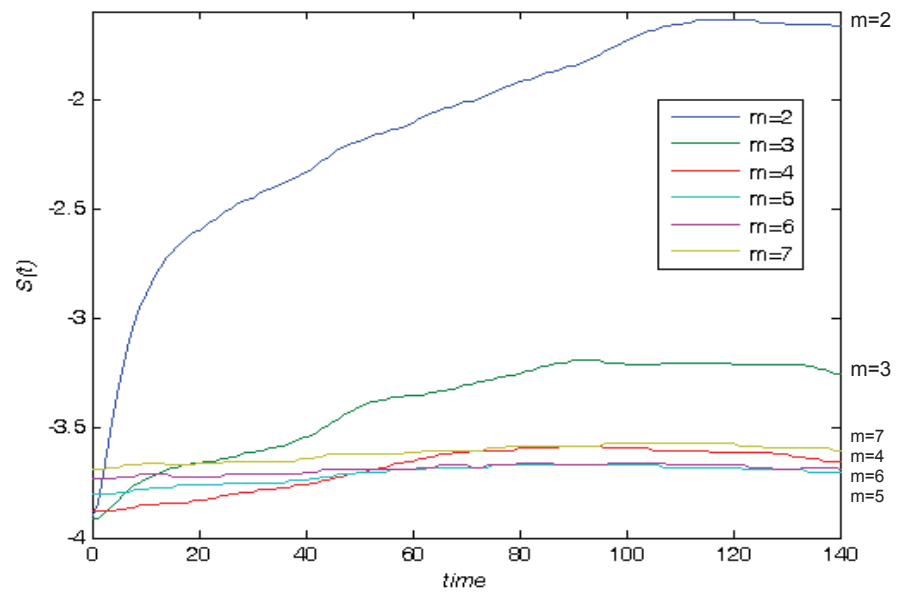

Figure 10

The Plot of $S(t)$ versus Time of Expected Inflation Series

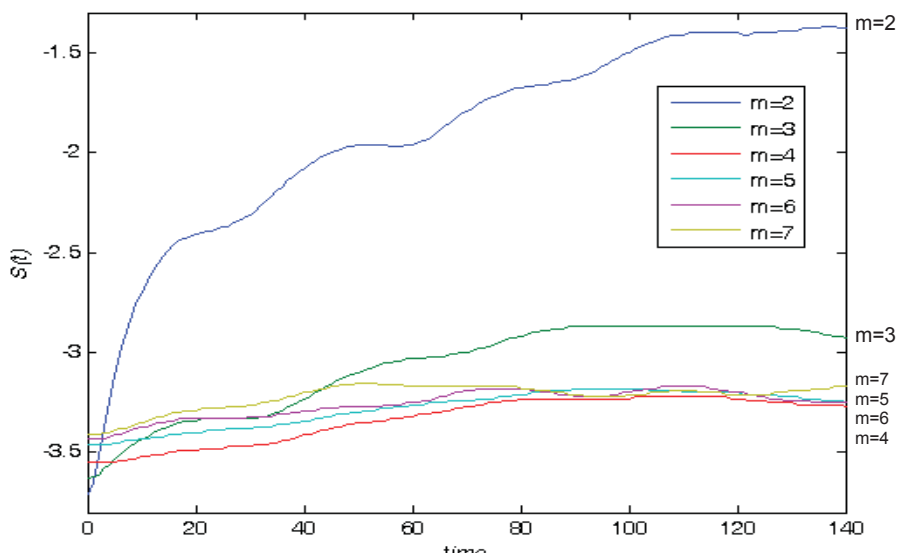


By the same token, we analyse the evolution of price level and expected inflation of the four-equation model. These time series are generated by the four-equation model calibrated in numerical example 2 in Section 5. When calibrating our four-equation model, we keep all parameters in the last two equations unchanged so that later we can observe how fluctuations in the real sector of economy affect the price level and expected inflation in the monetary sector. The chaotic behaviour of these variables will be again determined through values of the correlation dimension and the largest Lyapunov exponent.

The correlation sums of all the series of our interest are calculated with program TISEAN for embedding dimensions up to 10 and plots of the relation between correlation dimension and $\log (\varepsilon)$ in various embedding dimensions are shown in Figures 11 to 12 . It is clear that there is a required plateau area in which the correlation dimension can be determined. The correlation dimensions of price level and expected inflation series obtained from our four-equation model are calculated from the scaling region, as mentioned before, where the value fluctuates again in interval $(2,3)$ and we can make a similar conclusion about the correlation dimension of the structure of the system generated by four differential equations as in the case of the system of two equations. (The exact values of the correlation dimension of series price level and expected inflation are 2.3 and 2.6, respectively.)

Figure 11

Correlation Dimension vs. Log $(\varepsilon)$ of Price Level Series

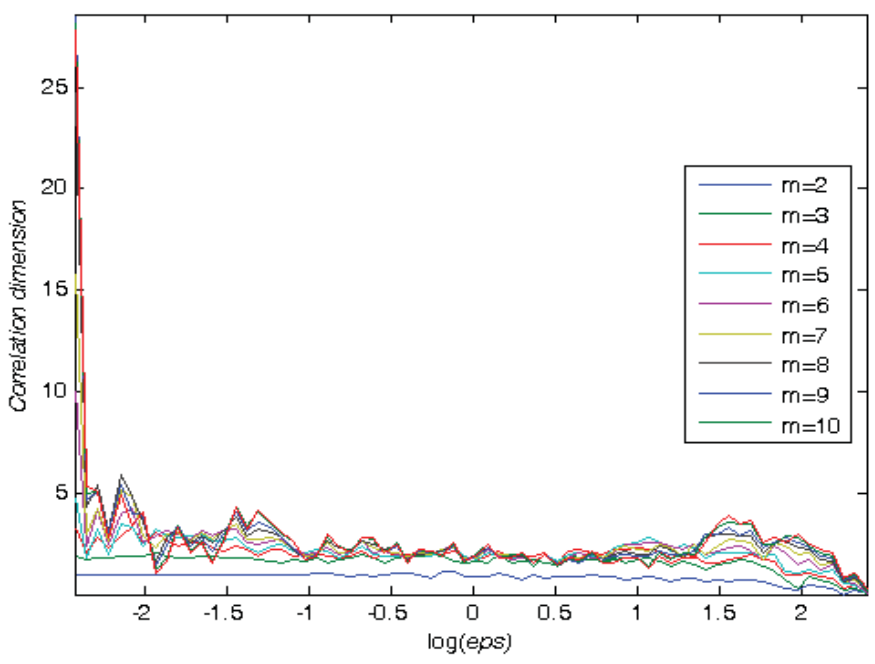


Figure 12

Correlation Dimension vs. Log $(\varepsilon)$ of Expected Inflation Series

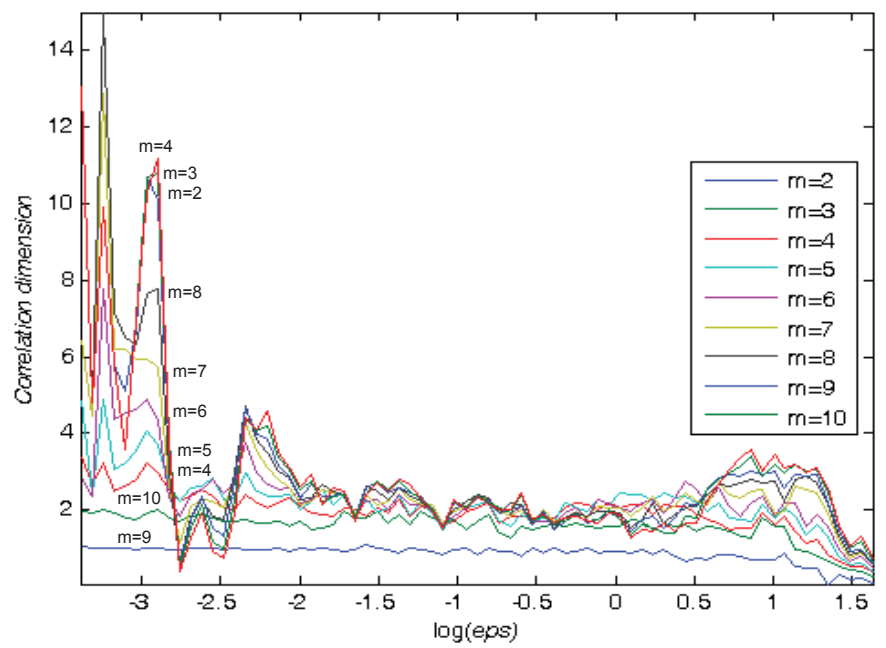

To calculate the largest Lyapunov exponent in series of our interest, it is necessary to determine suitable values of the time delay $\tau$ and the embedding dimension $m$. Time delay $\tau$ for these series are 26, where their mutual information measure reaches their first minimum. The chosen embedding dimensions for estimation of the largest Lyapunov exponent are $m=2,3,4$ and 5. The values of $S(t)$ are calculated by program TISEAN again. The plots of $S(t)$ versus $t$ for series of our interest are shown in Figures 13 to 14 . The value of the largest Lyapunov exponent of price level series equals 0.15 and the one of expected inflation series equals 0.10 .

Comparing the estimated values of correlation dimension and the largest Lyapunov exponent of dynamic system of price level and expected inflation of the two-equation model with the one of the four-equation model, it can be seen that the introduction of the fluctuations of the real sector of economy only leads to a slight change in the geometrical structure of trajectories of our chaotic system in state space. Namely, the evaluated correlation dimension of the system has changed downwards slightly from 2.5 to 2.8 to 2.3 to 2.6 , respectively. On the other hand, as far as the dynamics of our chaotic behaviour is concerned, the involvement of fluctuations of the real sector of economy causes a substantial change in the dynamics of the examined chaotic system. The estimated values of the largest Lyapunov exponent increases from 0.8 and 0.02 to 0.15 and 0.10 , respectively. It is necessary to point out that in both cases the value of the largest Lyapunov exponent of our system is rather small. 
Figure 13

The Plot of $S(t)$ versus Time for Series of Price Level in 4-Equation Model

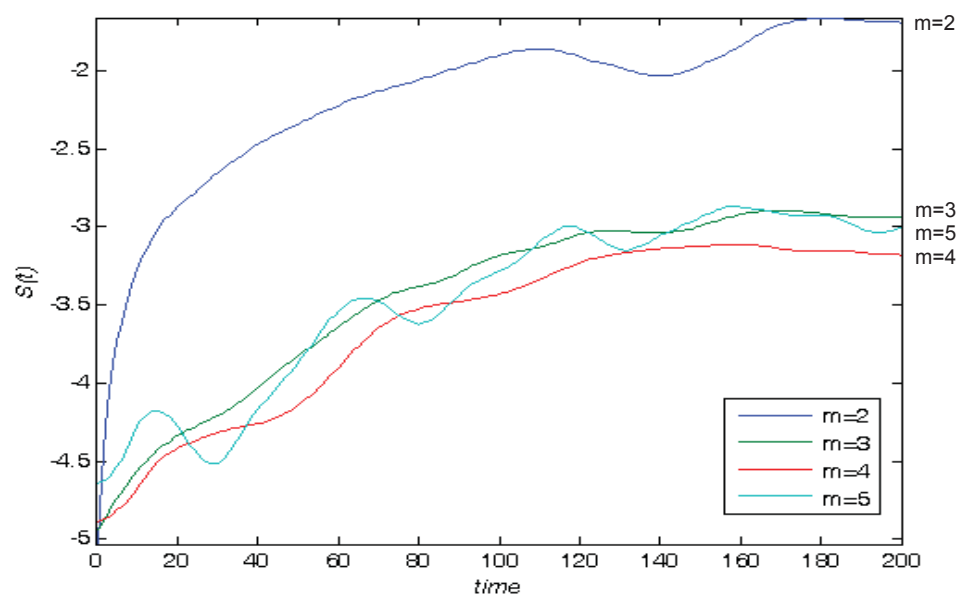

Figure 14

The Plot of $S(t)$ versus Time for Series of Expected Inflation in 4-Equation Model

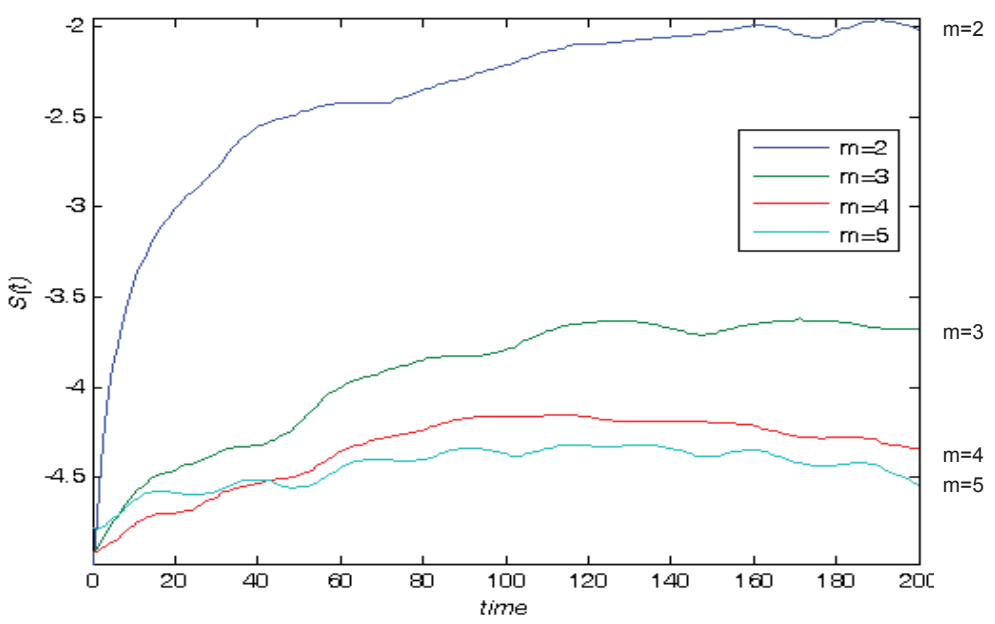

\section{Conclusion}

In this article we have tried to find an answer to the question if traditional models of inflation can produce complex dynamics. In traditional linear models, economic variables can only exhibit linear fluctuations, which is in sharp contrast to behaviour of actual economic variables which very often produce non-periodical oscillations. Trying to explain this behaviour, we use non-linear deterministic approach where the complex dynamics stems from endogenous structure of system, i.e. from its non-linearity. First, 
we introduced a two equation model to explain the dynamics of price level and expected inflation in an economy. Then we add Kaldor's model which describes the dynamics of capital and production in the economy to the two equation model to connect oscillations in real economy to the dynamics of price level and expected inflation. By combining Kaldor's model with the two equation model of price dynamics mentioned we get our so called four equation model which contains two segments: the real segment captured by Kaldor's model and the second one captured by our two equation model of price dynamics. This connection is not only possible, but thank to interaction of the two segments, it makes the dynamics of the whole system more complex.

To analyse behaviour of variables of our interest in the model, a set of desirable parameters is chosen for the two equation model and a time series of price level and expected inflation is generated. Using state space reconstruction technique, we estimate the values of two typical descriptors of chaotic systems from our time series generated by the two equation model: correlation dimension and the largest Lyapunov exponent. For our series we find that the value of the correlation dimension of the attractor is 2.5 . The value of the largest Lyapunov exponent is 0.08 which is a positive but relatively small. The fact that correlation dimension of the system is constant at 2.5 with positive largest Lyapunov exponent indicates that nonlinear technique can detect and identify the existence of deterministic chaos in our system generating the time series used for our analysis.

In our four equation model the output of our numerical example shows that merging the two equation model with Kaldor's model is possible and makes the dynamics of the whole system more complex. First, the simple dynamics of two variables product and capital still evolves in line with the theory. Then, using the same set of parameters for two equation model, we introduce oscillations in the real sector of economy and connect them with the price dynamics and generate a time series of price level and expected inflation. Again, we use the state space reconstruction technique to determine correlation dimension and the largest Lyapunov exponent of the reconstructed state space in the series generated by our four equation model. By introducing fluctuations in the real sector to the monetary one, the geometrical structure of trajectories of our system has changed slightly, its correlation dimension increases from 2.5 to 2.8 and the largest Lyapunov exponent, representing its dynamics, increases from 0.8 and 0.02 to 0.15 and 0.10 respectively as our non-linear technique analysis has detected. On the other hand, the deterministic chaos nature of the system is proved to be preserved.

Our results have confirmed our a priori expectation that including some simple non-linearity into traditional models can lead to more complex dynamical behaviour of these models. The confirmation of this idea has been a quite important for economic modelling because it solidifies the foundation for further development of modelling dynamic systems which are based on traditional macro-economic theory. As such, it shows that deterministic models are also able to capture the realistic movement of macroeconomic variables. We also show that non-linear time series technique can successfully 
detect and quantify the presence of deterministic chaos in time series generated by a dynamic system and once when the availability of real data is not an issue, one can look into the data and examine the possible existence of chaos in real economic processes. For further research but it would be very interesting to investigate how the model could behave under bifurcation.

\section{References}

Allen, R. D. G. (1956), Mathematical Economics. London: Macmillan.

Arlt, J., Kodera, J., Mandel, M., Tomšík, V. (2006), "Monetary Approach to Inflation: A Medium Term Structural Model in a Small Open Economy." Politická Ekonomie, Vol. 54, No. 3, pp. 326-338.

Chiarella, C. (1990), The Elements of a Nonlinear Theory of Economic Dynamics. Berlin: Springer Verlag.

Flaschel, P., Franke, R., Semmler, W. (1997), Dynamic Macroeconomics. Cambridge, Massachusetts: MIT Press.

Friedman, M. (1956), "The Quantity Theory of Money-A Reastatement." In "Studies in Quantity Theory of Money." Chicago: The University of Chicago Press.

Frisch, R. (1933), "Propagation Problems and Impulse Problems in Dynamic Economics." In Allen and Unwin (eds.): Essays in Honor of Gustav Cassel. London.

Gabisch, G., Lorenz, H. W. (1989), Business Cycle Theory. Berlin: Springer Verlag.

Goodwin, R. M. (1951), "The Non-Linear Accelerator and Persistence of Business Cycle." Econometrica, Vol.19, No.1, pp. 1-17.

Guckenheimer, J., Holmes, P. (1986), Non-Linear Oscillation, Dynamical Systems and Bifurcations of Vector Fields. New York: Springer Verlag.

Hegger, R., Kantz, H., Schreiber, T. (1999), "Practical Implementation of Non-linear Time Series Methods: The TISEAN Package", CHAOS 9.

Horák, J., Krlín, L., Raidl, A. (2003), Deterministický chaos a jeho fyzikální aplikace. Praha: Academia.

Kaldor, N. (1940), "A Model of the Trade Cycle." Economic Journal, 50, pp. 78-92.

Kalecki, M. (1971). Selected Essays on the Dynamics of the Capitalist Economy, 1933-1970. Cambridge: Cambridge University Press.

Kantz, H., Schreiber, T. (1997), Non-Linear Time Series Analysis. Cambridge: Cambridge Nonlinear Science Series.

Kodera, J., Vošvrda, M. (2006), "Product, Capital and Price Dynamics in the Simple Model of Closed Economy (Produkt, kapitál a cenový pohyb v jednoduchém modelu uzavřené ekonomiky)." Politická ekonomie Vol. 54, No. 3, pp. 339-350.

Kodera, J., Sladký, K., Vošvrda, M. (2003), “The Extended Kalecki-Kaldor Model Revisited," In Proc. 21th Internat. Conference Mathematical Methods in Economics (M. Houška, ed.), Faculty of Economics, Czech University of Agriculture, Praha, pp. 160-165.

Kodera, J. (2002), "Four Equation Model of Price Dynamics." In Proc. 20th Internat. Conference Mathematical Methods in Economics, University of Economics, Ostrava, pp. 151-155.

Kuznetsov, Y. A. (1998), Elements of Applied Bifurcation Theory. Berlin: Springer Verlag.

Kydland, F. E., Prescott, E. C. (1982), "Time to Build and Aggregate Fluctuations." Econometrica 50 (6): pp. 1345-1370.

Lorenz, H. W. (1994), Non-Linear Dynamical Economics and Chaotic Motion. Berlin: Springer Verlag.

Perko, L. (2001), Differential Equations and Dynamical Systems. Berlin: Springer Verlag.

Phillips, A. W. (1954), "Stabilisation Policy in a Closed Economy." Economic Journal 64, pp. 290-323. 
Rosser, J. B. Jr. (1999), "On the Complexities of Complex Economic Dynamics." The Journal of Economic Perspectives, Vol. 13, No. 4, pp. 169-192.

Sprott, J. C. (2003), Chaos and Time-Series Analysis. Oxford: Oxford University Press.

Takens, F. (1985), "On the Numerical Determination of the Dimension of an Attractor." In Braaksma, B. L. J., Broer, H. W., Takens, F., eds., Dynamical Systems and Bifurcations. Lecture Notes in Math, Heidelberg: Springer Verlag.

Tinbergen, J. (1930), "Ein Schiffsbauzyklus?" in Weltwirtschaftliches Archiv, reprinted in Klaasen, L. H. et al. (1959), ed., Selected Papers, Amsterdam. 